\title{
Claus Claussen - ein Wegbereiter der modernen Radiologie
}

Mit Prof. Dr. med. Claus D. Claussen verlässt im März 2014 einer der renommiertesten radiologischen Lehrstuhlinhaber die Bühne der universitären Medizin. Seine Emeritierung nach mehr als 25 Jahren ist Anlass genug, die akademische Vita, die zukunftsweisenden Ideen und das berufspolitische Engagement von Herrn Claussen aus der Sicht eines jahrelangen Wegbegleiters Revue passieren zu lassen.

In der akademischen Radiologie hat Herr Claussen außerordentlich viel erreicht - Promotion 1971 (Ruprecht-Karls-Universität Heidelberg), Habilitation 1982 (Freie Universität Berlin), C3Professur für Radiologie 1986 (Freie Universität Berlin), Berufung auf die C4-Professur für Radiologie als Nachfolger des renommierten Lehrstuhlinhabers Prof. Frommhold an der Eberhard Karls Universität Tübingen 1988 und Ernennung zum Visiting Professor der Mayo Clinic in Rochester/U.S. A. Stets zählte er zu den Ersten, die seit Mitte der 70er-Jahre innovative radiologische Verfahren wie die CT, MRT, MehrzeilenCT, PET-CT, PET-MRT, Laserangioplastie und den Einsatz medikamtentenbeschichteter Ballons zur PTA evaluiert haben. Als Wissenschaftler, Teamplayer und Mentor hat Herr Claussen viele junge Kolleginnen und Kollegen mit einer Kombination aus Herausforderung und Wohlwollen begleitet und seinen „Schülern“ Perspektiven bis hin zur Professur und zum Ordinariat eröffnet. Im produktiven Umfeld der Tübinger Abteilung, die Herr Claussen zu einem der Leuchttürme der deutschen Radiologie aufgebaut hat, sind mehr als 800 Publikationen entstanden, darunter nicht wenige in Radiology, Circulation, Cancer, Nature Medicine und dem New England Journal of Medicine.

Neben der Förderung des radiologischen Nachwuchses hat Herr Claussen in Tübingen Strukturen geschaffen, die für die akademische Radiologie durchaus Modellcharakter haben. So kam es auf sein Betreiben Mitte der 90er-Jahre zur Ausschreibung einer der ersten C3-Professuren für Experimentelle Radiologie in Deutschland. Mittlerweile widmet sich die Sektion für Experimentelle Radiologie in Tübingen unter Leitung von Prof. Schick sehr erfolgreich der Vernetzung von Grundlagenforschung und klinischer Radiologie. Ein weiterer Meilenstein mit Modellcharakter war die Etablierung einer Kooperation mit der medizintechnischen Industrie „auf Augenhöhe“ („Private-Public Partnership“). Seit Ende der 90er-Jahre sind daraus innovative Verfahren wie die Mehrzeilen-CT, Ganzkörper-MRT, HochfeldMRT, PET-CT und PET-MRT vom Labor bis zur klinischen Anwendung mitentwickelt worden. Die
Private-Public-Partnership war aber auch Eckpfeiler der Einwerbung kompetitiver Drittmittel über die DFG und das BMBF und bei der Etablierung einer Stiftungsprofessur durch die WernerSiemens-Stiftung aus der Schweiz. Damit konnte das Labor für präklinische Bildgebung und Bildgebungstechnologie eingerichtet und Herr Prof. Pichler als international ausgewiesener Wissenschaftlicher gewonnen werden.

Auch berufspolitisch hat sich Herr Claussen über viele Jahre für die Radiologie engagiert. Die Gründung der Konferenz der Lehrstuhlinhaber für Radiologie (KLR), deren Vorsitzender er von 1991 1997 war, ging wesentlich auf seine Initiative zurück. Von 1997 - 2009 war er im Vorstand der Deutschen Röntgengesellschaft, davon 2001 2003 als DRG-Präsident. Während dieser Zeit hat Herr Claussen gemeinsam mit Kollegen wie Prof. Kauffmann und Prof. Mödder die Weichen für eine Professionalisierung der DRG gestellt und Herrn Lewerich als Geschäftsführer rekrutiert. Später folgte der Umzug der DRG-Geschäftsstelle nach Berlin, wofür Herr Claussen sich in der festen Überzeugung sehr engagiert hat, dass eine wissenschaftliche Fachgesellschaft wie die DRG auch die räumliche Nähe zu wichtigen Entscheidungsträgern der Politik braucht. Während der Restrukturierungsphase des European Congress of Radiology (ECR) bzw. der European Association of Radiology (EAR) zur European Society of Radiology (ESR) hat Herr Claussen eine nicht unwesentliche Rolle gespielt. Aktuell ist er in den neu entstandenen Strukturen der ESR Vorsitzender des Subcommittee of Ethical Compliance. Als Kongresspräsident der ESMRMB (1988), der ESGAR (1999), des Deutschen Röntgenkongresses (2009) und Veranstalter vieler Symposien hat Herr Claussen organisatorisches Geschick und ein untrügliches Gespür für die wesentlichen Details bewiesen.

Neben seinem Engagement für die deutsche und europäische Radiologie hat Herr Clausen auch dem akademischen Geschehen an der Eberhard Karls Universität Tübingen große Aufmerksamkeit gewidmet. Er war von 1998 - 2000 Prodekanund von 2000 - 2006 über drei Wahlperioden Dekan der Medizinischen Fakultät. Unter seiner Ägide als Dekan wurde u. a. ein objektives System zur Bewertung wissenschaftlicher Leistungen zwischen den verschiedenen Fachgruppen der Fakultät erarbeitet und umgesetzt. Herr Claussen war darüber hinaus von 1998 - 2010 Mitglied des Senats der Eberhard Karls Universität Tübingen und gehörte von 2000 - 2004 der Grundsatzkommission seiner Universität an. In Anerkennung seiner Verdienste haben ihn die Französische Röntgen- 
gesellschaft, die Österreichischen Röntgengesellschaft, die Deutschen Röntgengesellschaft und die ESGAR zum Ehrenmitglied ernannt.

Aber Herr Claussen ist nicht nur Radiologe und Wissenschaftler. Er ist vor allem ein Mensch, dem Familie und Freunde wichtig sind und dessen Interessen der Kultur, die er vielfältig gefördert hat, der Politik und dem Sport gelten. Daraus sind Freundschaften zu den unterschiedlichsten Menschen entstanden, mit denen Herr Claussen einen Opernbesuch in Stuttgart genauso teilen kann wie das Pokalfinale im Berliner Olympiastadion. Er ist überzeugter Tübinger, aber dennoch Weltbürger und bekennender Wahlberliner. Seine facettenreiche Persönlichkeit vereint so vermeintlich verschiedene Eigenschaften wie Disziplin und Libera- lität, professionelles Selbstbewusstsein und Selbstironie, mutiges Durchsetzungsvermögen und kooperativer Führungsstil. Was mich darüber hinaus persönlich immer für Herrn Claussen eingenommen hat, sind sein sportlich-jugendliches Auftreten, sein Humor und vor allem eine beispiellose Großzügigkeit, die ich in den vielen gemeinsamen Jahren immer wieder erlebt habe. Meine allerbesten Wünsche und die Gewissheit auf ein Fortbestehen unserer mehr als 30jährigen Freundschaft begleiten Herrn Claussen in den neuen Lebensabschnitt, in dem wahrscheinlich weitaus mehr als nur der Ruhestand auf ihn wartet.

Prof. Dr. Michael Laniado, Dresden 